NBER WORKING PAPER SERIES

\title{
THE EFFICIENCY OF DECENTRALIZED INVESTMENT \\ MANAGEMENT SYSTEMS
}

David S. Jones

Working Paper No. 119

\section{NATIONAL BUREAU OF ECONOMIC RESEARCH \\ 1050 Massachusetts Avenue \\ Cambridge MA 02138}

July 1981

The research reported here is part of the NBER's research program in Financial Markets and Monetary Economics. Any opinions expressed are those of the author and not those of the National Bureau of Economic Research. 


\section{ABSTRACT}

The primary purpose of this paper is to demonstrate that decentralized investment management systems may not always be efficient. Specifically, within the context of a particular portfolio choice paradigm it is shown that a given decentralized investment management system is (weakly) efficient if and only if the joint probability distribution of asset rates of return satisfy certain covariance restrictions. If these restrictions do not obtain then the asset portfolios generated by this decentralized structure will generally be inferior to those which would be generated by a completely centralized structure.

This paper also discusses how the managers of departments within an efficient decentralized structure should behave so as to generate portfolios which are optimal from the point of view of the institution as a whole. Generally, departmental managers should behave as if they have less risk aversion than the institution as a whole. In fact, a given manager should be more risk averse the greater the value of his portfolio.

Finally, we note that the efficiency concept employed in this paper is equivalent to the proposition that certain assets admit consistent simple sum aggregation. It is shown that this implies that the efficient decentralization of investment decisions permits the institution to economize on the information which must be passed to higher level departments.

David S. Jones Department of Economics Northwestern University Evanston, Illinois 60201 
THE EFFICIENCY OF DECENTRALIZED INVESTMENT MANAGEMENT SYSTEMS

By David S. Jones

$\underline{\text { Introduction }}$

Most large institutional investors have some form of decentralized investment management system. Many of these systems take forms similar in spirit to the hierarchical structure depicted in Figure 1. At each tier in this structure a department committee or manager allocates the funds entrusted to it among various subordinate departments. When investible funds reach one of the departments along the bottom rung they are directly invested into the individual assets under the purview of that department. The solid lines in Figure 1 delineate the alternative channels by which investible funds flow through the institution.

The departments in a decentralized investment management system also tend to be informationally segmented in the following sense: each department formulates its investment decisions solely on the basis of its subjective assessment of the joint probability distribution of the rates of return of the assets under its purview. Information concerning the investment decisions or opportunities of departments specializing in other assets is either not available or is not used.

In the present paper we will discuss the conditions under which decentralized investment management systems of the above type are efficient. Before defining the concept of efficiency that we shall employ in this paper it will be useful to first describe the portfolio investment paradigm which underlies our analysis.

We shall assume that the investing institution as a whole has a 
$-2-$

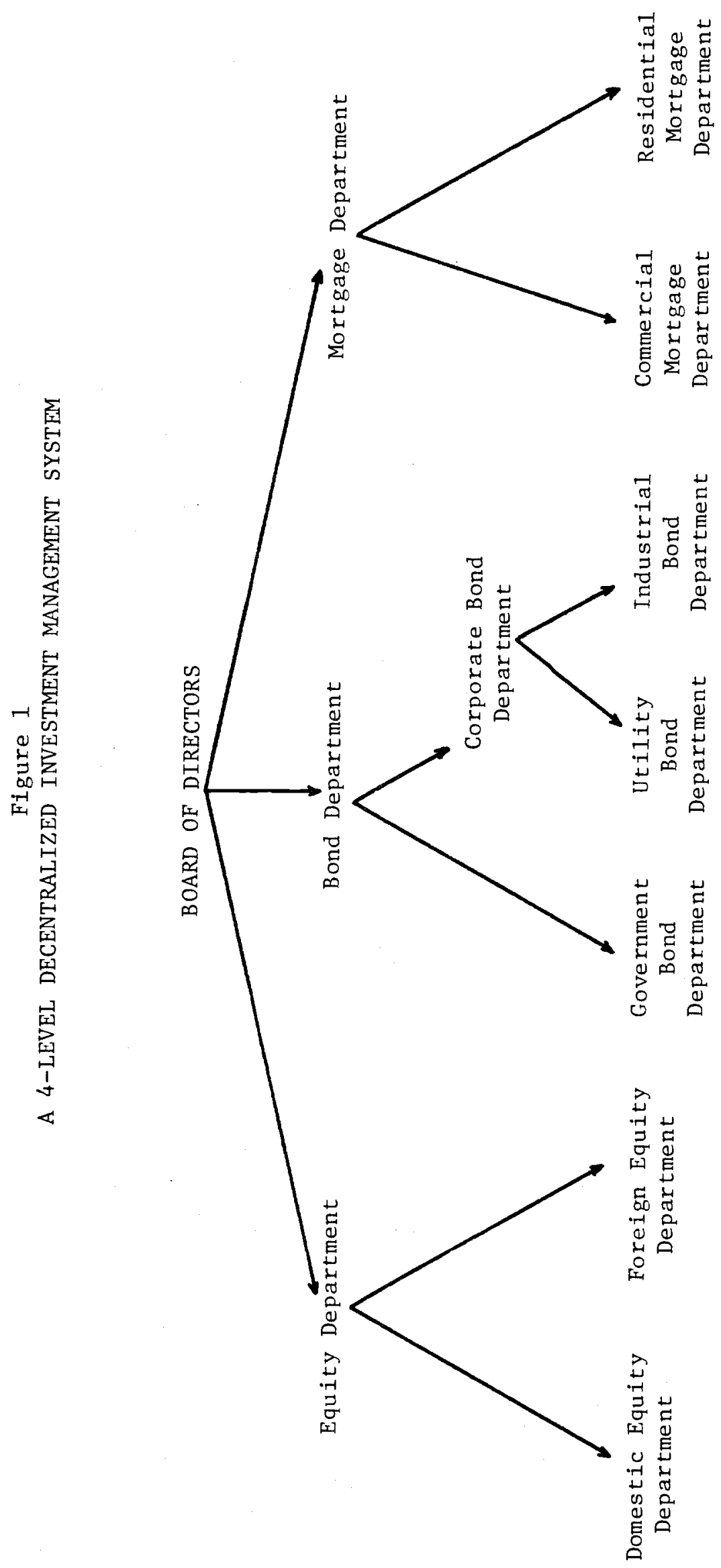


certain amount of funds to allocate at the beginning of a period. It desires to allocate these funds among assets so as to maximize the expected utility of its end-of-period wealth. $1 /$ Transactions costs associated with the purchase and sale of assets are assumed to be nonexistent.

Our analysis also abstracts from information costs. That is, the costs of acquiring and disseminating information about future asset rates of return are taken to be zero. We also assume that the subjective joint probability distributions of rates of return employed by the various departments are consistent with one another. Thus, in Figure 1, the board of directors, the equity department, and the domestic equity department all have the same subjective joint probability distribution of rates of return for domestic equities.

Within the context outlined above, the efficiency concept that we have in mind is as follows: a given investment management structure is efficient if for all information sets it gives rise to the same portfolio allocation as would a completely centralized scheme in which all individual investment decisions are made by the board of directors. Since we are abstracting from information and transactions costs it is clear that there is no reason for a decentralized structure to be preferred to a completely centralized structure. Hence, the best a decentralized structure could hope to accomplish is to replicate the investment decisions of a completely centralized structure with the same information.

Since the existence of information costs is presumably a major reason why decentralized investment management structures are adopted in practice, the reader may find it curious that we choose to abstract from them here. $\frac{2}{}$ 
We do so in order to highlight as simply as possible an innate problem with decentralized investment structures. To anticipate, such schemes will normally generate inefficient portfolio allocations because the informational segmentation inherent in these structures does not permit individual departments to accurately assess the effects of their individual actions on the "riskiness" of the institution's overall portfolio. II. The Paradigm of Investor Behavior

Consider an institutional investor with wealth $\mathrm{W}_{0}$ to be allocated among " $k$ " assets at the beginning of a period. The institution desires to allocate this wealth so as to maximize a utility function defined in terms of the mean and variance of its portfolio's rate of return for the period. $\underline{3} /$ That is, its objective is to choose the $(k \times 1)$ portfolio share vector $\underline{h}$ which maximizes the utility function

$$
\mathrm{u}\left(\mu, \sigma^{2}\right) ; \mathrm{u}_{1}>0 ; \mathrm{u}_{2}<0
$$

where $\mu=$ the subjective mean of the portfolio's real rate of return and $\sigma^{2}=$ the subjective variance of the portfolio's real rate of return. Denote the $(k \times 1)$ vector of expected rates of return on the " $k$ " alternative assets by $\underline{\underline{r}}$ and denote the associated variance-covariance matrix by $\Omega .4 /$ Then the investor's portfolio choice problem can be formulated mathematically as the following constrained maximization problem:

$$
\begin{array}{ll}
\underset{\operatorname{maximize}}{\mathrm{h}} & \mathrm{u}\left(\underline{h}^{\mathrm{T}} \underline{\underline{r}}, \underline{h}^{\mathrm{T}} \Omega \underline{h}\right) \\
\text { subject to: } & \underline{1}^{\mathrm{T}} \underline{\mathrm{h}}=1
\end{array}
$$

where $\underline{1}$ is an appropriately dimensioned column vector of ones.

For simplicity, in the analysis below we shall presume that the meanvariance utility function $u\left(\mu, \sigma^{2}\right)$ takes the particular form: 


$$
u\left(\mu, \sigma^{2}\right)=\mu-(\rho / 2) \sigma^{2}
$$

where the parameter " $\rho$ " is the investor's relative risk aversion with respect to wealth. While, in general, this parameter may vary with respect to the other parameters of the problem, for simplicity, we shall take " $\rho$ " to be a constant below. That is, we shall assume that the investor displays constant relative risk aversion. Thus, problem (2) becomes

$$
\begin{aligned}
& \text { maximize } \quad \underline{\mathrm{h}} \underline{\mathrm{T}} \underline{\underline{r}}-(\rho / 2) \underline{h}^{\mathrm{T}} \Omega \underline{\mathrm{h}} \\
& \text { subject to: } \underline{1}^{\mathrm{T}} \underline{\mathrm{h}}=1 \text {. }
\end{aligned}
$$

The specific utility function given above can be motivated or justified in a number of ways. It can be interpreted as a first-order Taylor series approximation to an arbitrary mean-variance utility function. Alternatively, Friend, Landskronner, and Losq [1976] and Friedman and Roley [1979] have shown that, under certain conditions, as holding periods become short the solution to a constant relative risk aversion von Neumann-Morgenstern expected utility (in terms of end-of-period wealth) maximizer's portfolio choice problem tends to the solution to problem (8). Jones [1979] has also shown that Lintner's [1972] lognormal securities market model of investor behavior tends to (3) as the conditions needed for Lintner's approximations to be exact are approached. Finally, under general conditions Merton's [1973] continuous-time asset trading model for an investor with constant relative risk aversion can be shown to imply that the investor continuously solves the instantaneous portfolio choice problem (3) if $\overline{\underline{r}}$ and $\Omega$ are interpreted as the instantaneous mean vector and variance-covariance matrix of a Gaussian diffusion process which generates actual rates of return. 
III. The Efficiency of 2-Level Investment Management Systems

Before investigating the efficiency of multilevel management

structures like the 4-level structure depicted in Figure 1, it will prove to be convenient to first examine the efficiency of 2-1evel structures like that illustrated in Figure 2. To this end, partition the portfolio share vector $\underline{h}$ into the "s" asset groups implicit in the structure of Figure 2 . Also, partition $\underline{\underline{r}}$ and $\Omega$ conformably. Hence,

$$
\underline{\mathrm{h}}=\left(\underline{\mathrm{h}}_{1}, \ldots, \underline{\mathrm{h}}_{\mathrm{s}}\right)^{\mathrm{T}} ; \underline{\underline{\mathrm{r}}}=\left(\underline{\underline{r}}_{1}, \ldots, \overline{\mathrm{r}}_{\mathrm{s}}\right)^{\mathrm{T}} ; \text { and } \Omega=\left[\begin{array}{c}
\Omega_{1,1} \cdots \Omega_{1, s} \\
\Omega_{s, 1} \ldots \Omega_{s, s_{-}}
\end{array}\right] \text {. }
$$

It is straightforward to show that the portfolio share vector which solves problem ( 3$), \underline{h}^{*}$, is given by:

$$
\underline{h}^{*}=(1 / \rho) B \underline{\bar{r}}+\underline{b}
$$

where $\mathrm{B} \equiv\left[\Omega^{-1}-\left(1 / \underline{\underline{1}}^{\mathrm{T}} \Omega^{-1} \underline{1}\right) \Omega^{-1} \underline{1} \underline{1}^{\mathrm{T}} \Omega^{-1}\right]$ and

$$
\underline{b} \equiv\left(1 / \underline{1}^{\mathrm{T}} \Omega^{-1} \underline{1}\right) \Omega^{-1} \underline{1} .
$$

By definition the optimal fraction of the investor's wealth allocated to the $i^{\text {th }}$ group of assets is $\underline{1}^{T} \underline{h}_{i}^{*}$. Thus, the optimal portfolio share vector for the $i^{\text {th }}$ department in the decentralized structure of Figure 2 is
(6)

$$
\left(1 / \underline{1}^{\mathrm{T}} \underline{h}_{i}^{*}\right) \underline{h}_{i}^{*}
$$

where we recall that this department is constrained to invest only in the assets under its purview (i.e., those of the $i^{\text {th }}$ asset group). Because of the informational segmentation implicit in the structure 


$$
-7-
$$
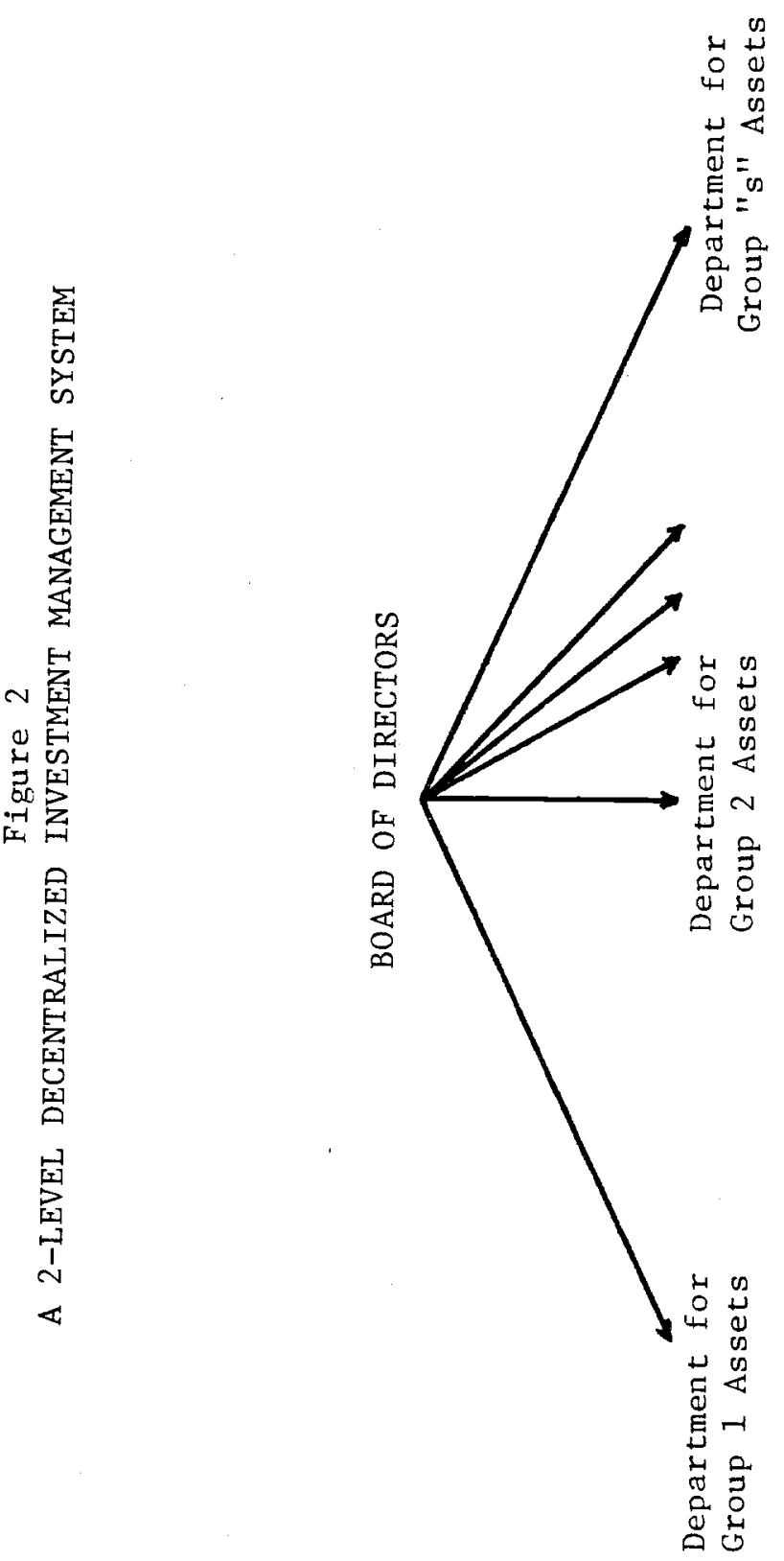
of Figure 2, the only information available to the $i^{\text {th }}$ department on which to base its investment decisions is its subjective assessment of the joint probability distribution of the rates of return of the $i^{\text {th }}$ group of assets. Hence, in order for the structure of Figure 2 to be efficient, it must be possible to express the quantity in (6) solely as a function of $\underline{\underline{r}}_{i}$ and $\Omega_{i, i}$. That is, a necessary and sufficient condition for (strong) efficiency is that there exist functions $\underline{g}_{\mathbf{i}}(.,$.$) such that$

(7) $\quad\left(1 / \underline{1}^{T} \underline{h}_{i}^{*}\right) \underline{h}_{i}^{*}=\underline{g}_{i}\left[\underline{\underline{r}}_{i}, \Omega_{i, i}\right]$ for $i=1, \ldots, s$

for all $\underline{\underline{r}}$ and $\Omega$ in the domain for which problem (3) is defined.

It is demonstrated in the appendix that g-functions satisfying the restrictions in (7) generally do not exist for $k>s>1$ unless the domains of $\underline{\bar{r}}$ and $\Omega$ are restricted. Consequently, a given decentralized investment management structure will generally only be efficient relative to a restricted class of $\overline{\bar{r}}$ and $\Omega$. We also show in the appendix that if $\underline{\bar{r}}$ is permitted to be unrestricted in $R^{k}$ then g-functions satisfying (7) for $k>s>1$ do not exist regardless of the restrictions on the domain of $\Omega$. Since there is in practice no a priori reason why expected yield vectors should be restricted to lie in a lower dimensional subspace of $R^{k}$, this result would seem to suggest that our present concept of (strong) efficiency is not very applicable to real world situations.

It turns out, however, that if we permit departments to have one additional bit of information, then we can find a limited class of $\Omega$ for which the structures in Figure 2 are efficient even if $\overline{\underline{r}}$ is unrestricted in $\mathrm{R}^{\mathrm{k}}$. The additional information that the $i^{\text {th }}$ department requires in order for this weaker efficiency to obtain is the fraction of the institution's total 
wealth allocated to the $i^{\text {th }}$ department (i.e., $\underline{1}_{{ }^{T}}^{\mathrm{T}}{ }_{i}^{*}$ ). Weak efficiency is then equivalent to the existence of functions $\underline{f}_{\mathbf{i}}(., .,$.$) such that$

$$
\left(1 / \underline{1}^{T} \underline{h}_{i}^{*}\right) \underline{h}_{i}^{*}=\underline{f}_{i}\left[\underline{r}_{i}, \Omega_{i, i}, \underline{1}^{T} \underline{h}_{i}^{*}\right] \text { for } i=1, \ldots, s
$$

for all $\underline{\bar{r}}$ and $\Omega$ in the appropriate domain.

In the appendix we provide the following theorem:

Theorem 1: Suppose the domain of $\underline{\underline{r}}$ is unrestricted. Then the largest class of nonsingular covariance matrices $\Omega$ for which the decentralized management structure in Figure 2 is (weakly) efficient is that in which the covariance matrices $\Omega_{i, j}(i \neq j)$ are of the form:

$$
\begin{aligned}
& \Omega_{i, j}=c_{i} \underline{1}^{T}-1 c_{j}^{T} i \neq j ; i, j=1, \ldots, s \\
& \text { where the } \underline{c}_{i} \text { are fixed vectors and the covariance matrices } \Omega_{i, i} \text { for }
\end{aligned}
$$
$i=1, \ldots, s$ are unrestricted. $\underline{6}$

Theorem 1 essentially states that the covariance matrices associated with the partitioned $\Omega$ matrix must assume the form in (9) in order for the management structure in Figure 2 to be efficient for all values of $\underline{\bar{r}}$. The covariance parameter vectors $\underline{c}_{1}, \ldots, c_{s}$ must be constant if the f-functions in (8) are to be stable functions of the $\underline{\bar{r}}, \Omega_{i, i}$, and $\underline{1}^{\mathrm{T}} \underline{h}_{i}^{*}$. As the sufficiency portion of the proof of Theorem 1 indicates, the function $\underline{f}_{i}$ is parameterized by $c_{i}$. Hence, unless the $\underline{c}_{i}$ are constant vectors the f-functions will not be stable in the above sense.

Several corollaries of Theorem 1 are readily apparent. First, if the rates of return of the assets in each group are uncorrelated with those of assets in all other groups, then the underlying 2-1evel management structure is (weakly) efficient. This corresponds to the case for which $\underline{c}_{i}=\underline{0}$ for a11 "i." 
Another special case occurs when there are two groups of assets and one of these groups contains a single asset. For such a partitioning of the assets the associated covariance matrix, $\Omega_{1,2}$, will always be of the form in (9). $\underline{\text { I }}$ Hence, a decentralized 2-level investment structure in which one department oversees a single asset while the other department oversees all the remaining assets is always (weakly) efficient.

Finally, another special case, of limited interest, obtains when there are " $k$ " departments and each department has responsibility over a single asset. Trivially, such a $2-1$ evel investment management structure is always efficient. $\frac{8}{}$ When condition (9) is satisfied the optimal portfolio share vector for the $i^{\text {th }}$ department in Figure 2 is given by:

$$
\begin{aligned}
& v_{i}^{*} \equiv\left(\underline{1}^{\mathrm{T}} \underline{h}_{i}^{*}\right) \underline{h}_{i}^{*}=\left(\frac{1}{\rho_{i}} \cdot \underline{1}^{\mathrm{T}} \underline{h}_{i}^{*}\right) \mathrm{B}_{i} \underline{\bar{r}}_{i}+\underline{b}_{i}-\frac{\left(1-\underline{1}_{h_{i}^{*}}^{*}\right)}{\underline{1}_{h_{i}^{*}}^{*}} \text { B }_{i} \underline{c}_{i} \\
& =\frac{1}{\left(\rho_{i} \underline{I}^{T} \underline{h}_{i}^{*}\right)} G_{i}\left(\bar{r}_{i}-f c_{i}\right)+\underline{g}_{i}
\end{aligned}
$$

where $B_{i}=\left[\Omega_{i, i}^{-1}-\left(1 / \underline{1}^{\mathrm{T}} \Omega_{i, i}^{-1}{ }^{-1} \Omega_{i, i}^{-1} \underline{1}^{\mathrm{T}} \Omega_{i, i}^{-1}\right]\right.$

$$
\begin{aligned}
\mathrm{b}_{i} & =\left(1 / \underline{1}^{\mathrm{T}} \Omega_{i, i}^{-1}\right) \Omega_{i, i}^{-1} \underline{1}, \\
G_{i} & \equiv\left[\Sigma_{i, i}^{-1}-\left(1 / \underline{1}_{\Sigma_{i, i}}^{\mathrm{T}}{ }^{-1} \Sigma_{i, i}^{-1} \underline{1} \underline{1}^{\mathrm{T}} \Sigma_{i, i}^{-1}\right],\right. \\
\underline{g}_{i} & \equiv\left(1 / \underline{1}^{\mathrm{T}} \Sigma_{i, i}^{-1} \underline{1}\right) \Sigma_{i, i}^{-1} \underline{1}, \text { and } \\
\Sigma_{i, i} & \equiv\left[\Omega_{i, i}-\underline{1} c_{i}^{\mathrm{T}}-\underline{c}_{i} \underline{1}^{\mathrm{T}}\right] .
\end{aligned}
$$

This expression, moreover, is easily seen to be the solution to the following mean-variance portfolio choice problem: 


$$
\begin{array}{ll}
\operatorname{maximize} & \underline{v}_{i}^{\mathrm{T}}\left(\overline{\underline{r}}_{i}-\rho \underline{c}_{i}\right)-\left(\rho_{i}^{*} / 2\right) \underline{v}_{i}^{\mathrm{T}} \Sigma_{i, i} \underline{\mathrm{v}}_{i} \\
\underline{v}_{i} & \underline{\underline{1}}^{\mathrm{T}} \underline{v}_{i}=1
\end{array}
$$

where $\rho_{i}^{*} \equiv \rho \cdot\left(\underline{1}^{\mathrm{T}} \underline{h}_{i}^{*}\right)$. Thus, when the investment management structure of Figure 2 is efficient, it is optimal for the manager of $i^{\text {th }}$ department to allocate his funds as if he is trying to solve the portfolio choice problem in (12). That is, he should act as if his relative risk aversion is $\rho_{i}^{*}$ and he is investing in a vector of assets with expected yield vector $\overline{\underline{r}}_{i}-\underline{c}_{i}$ and variance-covariance matrix $\Sigma_{i, i}$. Only then will his investment decision be optimal from the point of view of the institution as a whole.

There are two interesting aspects to the portfolio choice problem (12) and its solution (11). First, it is generally optimal for the department manager to act as if he has less relative risk aversion than the institution as a whole. This follows because $\rho_{i}^{*}<\rho_{i}$ in the likely event that $\underline{1}^{\mathrm{T}} \underline{h}_{i}^{*}<1$. In fact, the appropriate relative risk aversion for the manager increases linearly with the size of his department's portfolio: the more funds allocated to his department, the more risk averse he should act. The basic reason for this centers on the informational segmentation implicit in Figure 2. Since there is usually more than one department, the risk of a particular department's portfolio is being pooled with that of other departments. In particular, if the $\Omega_{i, j}$ of (9) are zero, then the $i^{\text {th }}$ department's contribution to the variance of the institution's overall portfolio is $\left(\underline{1}^{\mathrm{T}_{h}} \underline{-}_{i}^{*}\right){ }^{2} \cdot \operatorname{Var}\left\{\stackrel{\sim}{\mathrm{r}}_{i}\right\}$ where $\operatorname{Var}\left\{\tilde{r}_{i}\right\}$ is the variance of the real rate of return of the $i^{\text {th }}$ department's portfolio. Behaving in a less risk averse manner is one way in which a department manager can compensate for this pooling effect if he desires to 
focus upon the variance of his own portfolio in isolation. If $\mathrm{c}_{i}$ is collinear with 1 , then his optimal portfolio will equal the sum of the first two terms on the right-hand side of (11). 9 /

In general, covariances between rates of return of assets in different departments will not be so proscribed and allowances must be made for this fact by the department managers. This is why when $\underline{c}_{i}$ is not collinear with 1 it is optimal for the $i^{\text {th }}$ manager to behave as if he is investing in an artificial set of assets with mean vector and variance-covariance matrix given by $\overline{\underline{r}}-\rho \underline{c}_{i}$ and $\Sigma_{i, i}$ as in (12). His doing so essentially contributes the third term on the right-hand side of (11) to his optimal portfolio share vector. Notice that as $\underline{1}^{T} \underline{h}_{i}^{*}$ tends to unity or as $\underline{c}_{i}$ tends to a vector collinear with 1 this correction tends to zero.

When $\underline{c}_{i}$ is collinear with 1 then the $i^{\text {th }}$ department manager need not pretend that he is investing in artificial assets. His portfolio choice problem in this case is to:

$$
\begin{aligned}
& \text { maximize } \quad \underline{v}_{i}^{\mathrm{T}} \overline{\underline{r}}_{i}-\left(\rho{ }_{i}^{*} / 2\right) \underline{v}_{i}^{\mathrm{T}} \Omega_{i, i} \underline{v}_{i} \\
& \underline{\mathrm{v}}_{\mathrm{i}} \\
& \text { subject to: } \underline{\mathrm{v}}_{\mathrm{i}}^{\mathrm{T}} \underline{1}=1
\end{aligned}
$$

where $\overline{\underline{r}}_{i}$ and $\Omega_{i, i}$ are the "true" mean-vector and variance-covariance matrix of the assets under his purview. Notice, however, that he must still adjust (lower) his level of relative risk aversion relative to that of the institution as a whole to compensate for the pooling effect described above.

Before extending Theorem 1 to multilevel investment management structures like that in Figure 1, it is interesting to note that condition (9) is equivalent to the requirement that the assets in each group within Figure 2 
admit consistent simple sum aggregation. A similar duality between the conditions for decentralization and aggregation obtains in consumer choice theory. This duality relationship will prove useful in Section $V$ wherein we demonstrate that within an efficient management structure the information required by higher level departments in order to efficiently allocate funds to subordinate departments is only a small subset of the information available to these subdepartments. Efficient management systems thereby permit information flows between departments and subordinate departments to be economized.

IV. The Efficiency of Multilevel Investment Management Systems

We return now to the original focus of our study: to determine the conditions under which multilevel decentralized management structures, like that in Figure 1, are weakly efficient. An important result which we shall employ to this end is the following 1 emma:

Lemma 1: A given multilevel investment management structure, $\Gamma$, is efficient if and only if every 2-level structure formed by concatenating nonoverlapping departments and subdepartments of $\Gamma$ is efficient.

To illustrate the use of this lemma, consider the 4-1evel structure of Figure 1. All told, there are 12 2-1evel structures which can be formed by concatenating departments and subdepartments with mutually exclusive asset domains. These are displayed in Figure 3. Lemma 1 essentially says that the structure in Figure 1 is weakly efficient if and only if each of the 2-level structures in Figure 3 is weakly efficient.

The formal proof of Lemma 1 is tedious, but straightforward, and we shall 
Figure 3

THE 2-LEVEL STRUCTURES IMPLICIT IN THE 4-LEVEL INVESTMENT MANAGEMENT SYSTEM DISPLAYED IN FIGURE 1

1) Equities, Bonds, Mortgages

2) Equities, Bonds, Commercial Mortgages, Residential Mortgages

3) Equities, Government Bonds, Corporate Bonds, Mortgages

4) Equities, Government Bonds, Utility Bonds, Industrial Bonds, Mortgages

5) Domestic Equities, Foreign Equities, Bonds, Mortgages

6) Equities, Government Bonds, Corporate Bonds, Commercial Mortgages, Residential Mortgages

7) Equities, Government Bonds, Utility Bonds, Industrial Bonds, Commercial Mortgages, Residential Mortgages

8) Domestic Equities, Foreign Equities, Bonds, Commercial Mortgages, Residential Mortgages

9) Domestic Equities, Foreign Equities, Government Bonds, Corporate Bonds, Mortgages

10) Domestic Equities, Foreign Equities, Government Bonds, Utility Bonds, Industrial Bonds, Mortgages

11) Domestic Equities, Foreign Equities, Government Bonds, Corporate Bonds, Commercial Mortgages, Residential Mortgages

12) Domestic Equities, Foreign Equities, Government Bonds, Utility Bonds, Industrial Bonds, Commercial Mortgages, Residential Mortgages 
only outline it here. The "necessity" part of Lemma 1 derives from the fact that in our hypothesized world without transactions costs intermediate level departments in a multilevel structure (e.g., the equity department in Figure 1) function only to funnel the appropriate amounts of funds to their subordinate departments. If the multilevel structure is efficient then the portfolio of the institution as a whole would be invariant to whether funds are allocated by the board of directors to the subdepartments among the bottom tier of the structure directly or whether they are channeled to these subdepartments via intermediate departments. Hence, in order for a multilevel structure to be efficient the structures obtained from it by eliminating arbitrary intermediate and bottom level departments must also be efficient.

To prove the "sufficiency" portion of Lemma 1, simply note that if all 2-level structures implicit in a given multilevel structure are efficient, then any intermediate or bottom level department in the multilevel structure possesses sufficient information to allocate its funds directly to the individual assets under its purview. Hence, these intermediate departments certainly have enough information to optimally allocate funds to subordinate departments. Moreover, when funds reach a bottom level department, this department also has sufficient information to optimally allocate funds among individual assets. Hence, "sufficiency" obtains.

By employing Lemma 1 , one may verify the weak efficiency of any specific multilevel structure by using Theorem 1 to verify that each of the $2-1$ evel structures implicit in it is efficient. The weak efficiency of a given multilevel structure thus implies a set of covariance restrictions across asset rates of return. These restrictions are found by applying Theorem 1 repeatedly 
to the 2-level structures implicit in the multilevel structure. We have derived these implied covariance restrictions for the 4-level structure of Figure 1. Figure 4 summarizes these restrictions by displaying the required structure of the covariance matrix of rates of return. In this diagram underscores, "_," denote constant vectors and the $\alpha_{i, j}$ 's and $c_{i, j}$ 's are scalars. For comparison, in Figure 5 we display the covariance restrictions implied by the efficiency of the structure displayed in Figure 2. Notice that the efficiency of the multilevel structure in Figure 1 is a much more restrictive proposition than the efficiency of that in Figure 2. V. Efficiency and Consistent Simple Sum Aggregation Over Assets

As in the deterministic theories of production and consumer choice, there is an intimate relationship between the concepts of efficient decentralization and consistent simple sum aggregation. In order to explicate this relationship within the simplest context, let us first reexamine the generic 2-level decentralized structure of Figure 2. It will be recalled that, from Theorem 1, a necessary and sufficient condition for this structure to be efficient is that the variance-covariance matrix of asset rates of return satisfies the restrictions given in (9). In another paper, Jones [1980], the author has shown that (9) is also both necessary and sufficient for the universe of assets to admit consistent simple sum aggregation across each of the "s" asset groupings implicit in the structure of Figure 2. Hence, for a given 2-1evel decentralized structure, the notion of weak efficiency is essentially equivalent to the requirement that the universe of assets admit consistent simple sum aggregation with respect to the natural aggregation scheme implicit in the structure. 


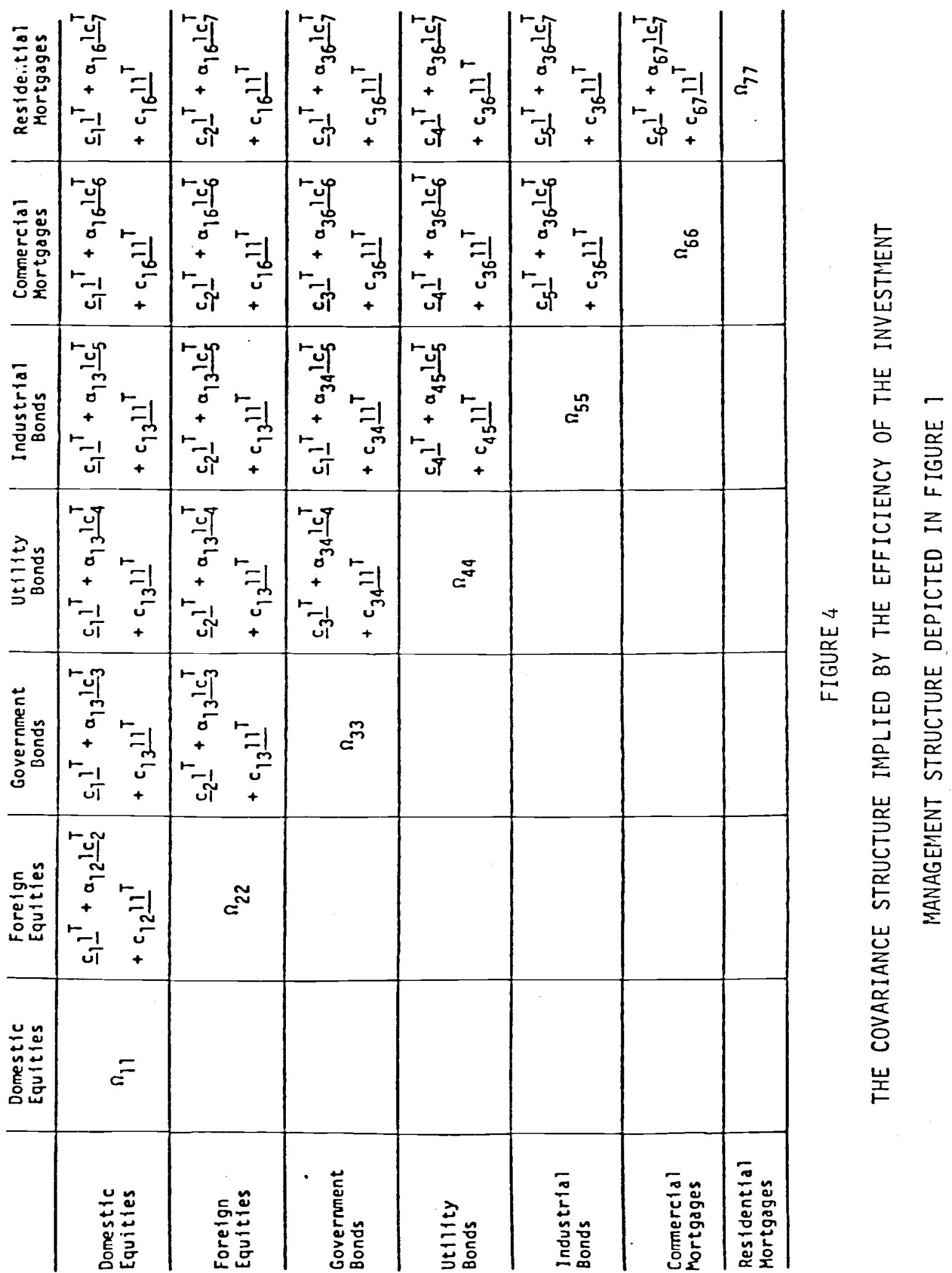




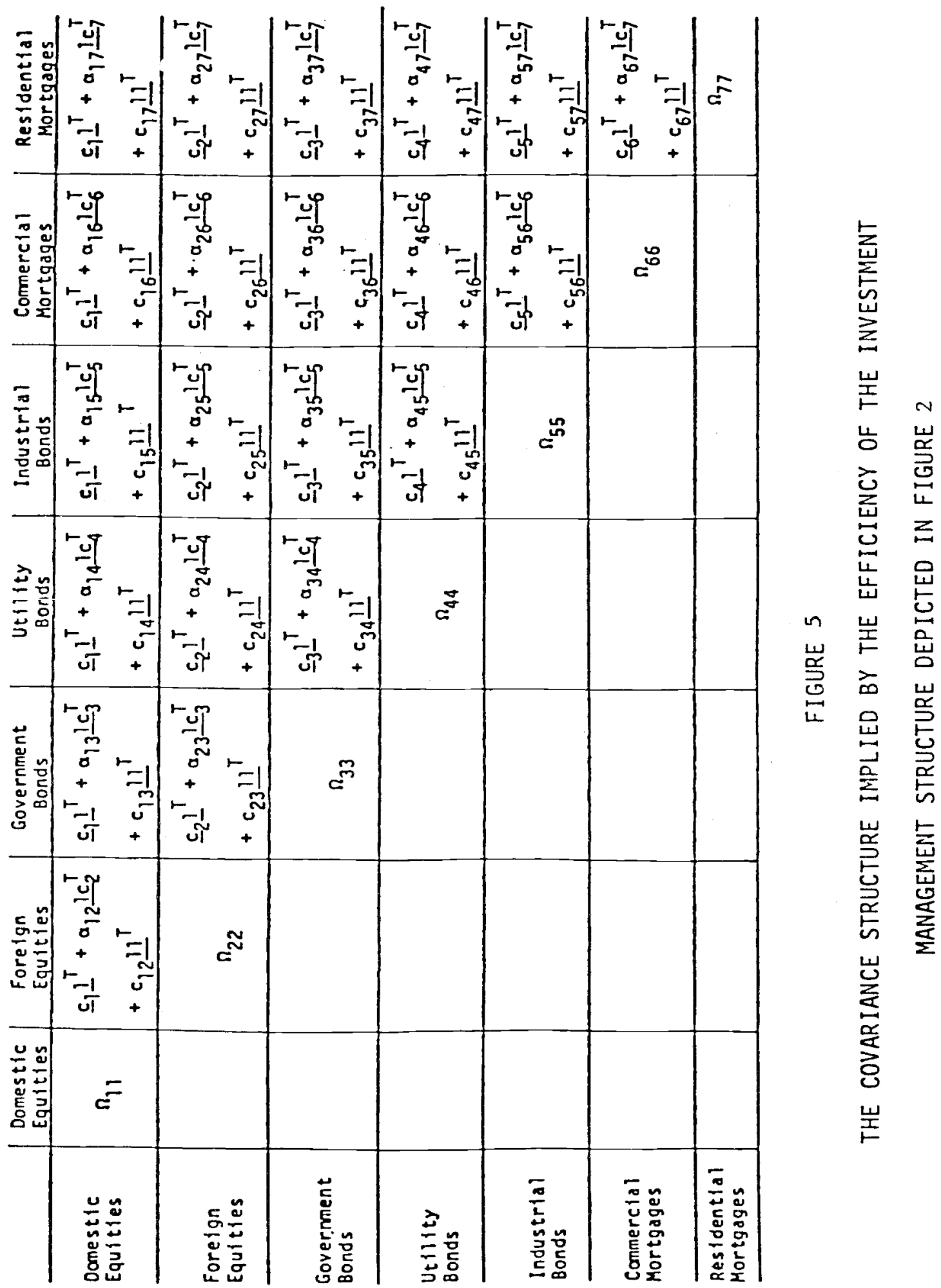


There is a natural extension of this result to multilevel management structures. To illustrate, consider the 4-level management structure displayed in Figure 1 and recall that its efficiency is equivalent to the proposition that each of the 2-level structures in Figure 3 is efficient. This implies that the structure in Figure 1 is efficient if and only if each of the simple sum aggregation schemes implicit in Figure 3 is consistent.

One useful implication of the above result concerns the choice of aggregation scheme to be employed in a portfolio choice model. Suppose that a multilevel management structure is efficient. Then all aggregation schemes formed from the 2-level structures implicit in the multilevel structure are consistent. That is, any one of them may be validly invoked. To a limited extent, then, a researcher is free to choose the aggregation scheme so as to simplify his task at hand.

The relationship between consistent simple sum aggregation and efficient decentralizability also has implications regarding the information which must be disseminated to departments in order for them to be able to efficiently allocate their funds to subordinate departments. As Jones [1980] makes clear, when a particular multilevel structure is efficient, it is not necessary for each department to know the joint probability distribution of yields on all assets within its purview. Rather, if the department's role is merely to allocate funds among subordinate departments then substantially less information will suffice. Specifically, this department can efficiently operate knowing only the joint probability distribution of the yields on as many "composite" assets as there are subordinate departments immediately below it on the organization chart. The allocation of funds among these subordinate 
departments then may proceed as if the department were itself directly investing funds among these few "composice" assets.

The yield index for the "composite" asset corresponding to the $i$ th subordinate department is constructed in the following manner: First, notice that efficiency implies that the covariance matrix between the $i^{\text {th }}$ and $j^{\text {th }}$ subordinate departments takes the form $c_{i} \underline{1}^{T}-\underline{1 c}_{j}^{T}$ for some vectors $\underline{c}_{i}$ and $\underline{c}_{j}$, where $c_{i}$ does not depend on "j." Next, define:

$$
G_{i}=\left[\Omega_{i, i}-\underline{c}_{i} \underline{1}^{T}-\underline{1 c}_{i}^{\mathrm{T}}\right]
$$

and

$$
\Sigma_{i}=\left[G_{i}^{-1}-\left(1 / \underline{1}^{T} G_{i}^{-1} \underline{1}^{-1} G_{i}{ }^{-1} \underline{11}^{T} G_{i}^{-1}\right]\right.
$$

where $\Omega_{i, i}$ is the variance-covariance matrix associated with the yields on the assets within the purview of the $i^{\text {th }}$ subordinate department. Then the yield index for this subordinate department, $\tilde{\phi}_{i}$, is given by:

$$
\tilde{\phi}_{i}=\underline{1}^{T}\left(\underline{r}_{i}-\rho \underline{c}_{i}\right) /\left(\underline{1}^{\mathrm{T}} \Sigma_{i} \underline{1}\right) \text {. }
$$

The reader should refer to Jones [1980] for a more detailed discussion and a derivation of this expression. VI. Summary and Conclusions

In this paper we have endeavored to demonstrate that decentralized investment management systems of the type described in section I are not always efficient. Specifically, within the context of the portfolio choice paradigm employed in this paper, it is shown that a given decentralized investment management system is (weakly) efficient if and only if the (subjective) joint probability distribution of asset rates of return satisfy certain covariance restrictions. If these covariance restrictions do not obtain then, from the point of view of the institution as a whole, the asset portfolios generated by this decentralized structure will generally be 
inferior to those which would be generated by a completely centralized structure.

This paper has also discussed how the managers of departments within an efficient decentralized structure should allocate their funds so as to maximize the "expected utility" of the institution as a whole. Generally it is optimal for these managers to act as if they have less risk aversion than the institution as a whole. In particular, the smaller is the dollar amount of funds controlled by a given manager, the less risk averse that manager should act.

Finally, we noted that the efficiency of a given decentralized investment management structure is equivalent to the proposition that the universe of assets admits consistent sum aggregation across certain asset groups. This result was shown to imply that the decentralization of investment decisions permits the institution as a whole to economize on the information which must be passed to higher level departments (e.g., the board of directors in our example) in order for them to be able to efficiently discharge their responsibilities.

Several shortcomings of the above analysis should be pointed out at this juncture. First, the analysis abstracts from information costs. It is likely that if such cost were included in the analysis, weaker conditions for a given decentralized investment management structure to be "economically efficient" would result.

Also, the generic decentralized investment structure probably presumes more informational and asset segmentation than is the case in real world institutions: Departments overseeing disjoint assets in the real world may 
exchange some information concerning each other's investment opportunities or investment decisions. Furthermore, in actual practice several departments may have the authority to invest in the same assets. For example, at some institutions, most department managers have the option of investing in a "cash" asset like deposits or Treasury bills. Efficiency for this latter type of structure has been partially analyzed by Jones [1979]. Therein, he derives a sufficient condition for the (weak) efficiency of 2-1eve1 investment management structures in which each department has the alternative of investing in a set of assets specific to that department and in a riskless asset which is the same across departments. This condition essentially replaces condition (9) of Theorem 1 with the requirement that $\left(9^{\prime}\right)$

$$
\Omega_{i, j}=0 \quad \text { for } \text { all } i \neq j ; i, j=1, \ldots, s \text {. }
$$


FOOTNOTES

*I wish to thank Vance Roley for his helpful comments on an earlier draft of this paper.

1/ While the actual utility function that we employ below is of the meanvariance variety, it is motivated within an expected utility maximization framework.

2/ A similar criticism may be directed toward the extensive literature on decentralizability or budgetability within the theories of consumer demand and production.

3/ Below, this paradigm is motivated within an expected utility maximization framework.

4/ In the ensuing discussions, $\Omega$ is always presumed to be nonsingular. This assumption may be motivated by postulating the absence of default free indexed bonds within an inflationary environment.

5/ Within the framework of Merton's [1973] saving-investment model this result obtains if unexpected changes in the vector of instantaneous mean rates of return is uncorrelated with the vector of unexpected actual instantaneous rates of return. The portfolio problem (3) can also be shown to result if the investor is assumed to have negative exponential von NeumannMorgenstern utility function defined in terms of portfolio real rates of return. 6/ As shown in Jones [1980], these covariance restrictions are essentially equivalent to the requirement that the objective function in (3) is separable in terms of the "s" asset groupings. In the language of Green [1964], this means that a two-stage budgeting. procedure would be consistent. 7/ This is because in this case $\Omega_{1,2}$ is one-dimensional. 
8/ This scheme is formally equivalent to a completely centralized management system in which the board of directors itself directly invests funds in individual assets.

g/ When $\underline{c}_{i}$ is collinear with $\underline{1}, B_{i} \underline{c}_{i}=\underline{0}$.

10/ To see how this fact economizes on information flows, consider a completely centralized investment management structure and the structures depicted in Figures 1 and 2. Suppose also that all are (weakly) efficient.

Within the completely centralized structure, the board of directors must have knowledge of $\underline{\bar{r}}$ and $\Omega$, involving a total of $k(3+k) / 2$ independent parameters. For $\mathrm{k}=100$ this number is 5150 .

Within the context of the structure in Figure 2, the board of directors requires knowledge of the mean vector and variance-covariance matrix associated with "s" composite assets. This involves only $s(3+s) / 2$ independent parameters. For $s=10$ this number is 65.

Within the context of the structure in Figure 1, the board requires knowledge of only nine independent parameters.

A similar economization of this information required by intermediate and bottom level departments can be shown to result as the investment management structure becomes more decentralized. 


\section{B IBL IOGRAPHY}

Friedman, B. and V. Roley, 1979, "A Note on the Derivation of Linear Homogeneous Asset Demand Equations," Nationa1 Bureau of Economic Research Working Paper No. 345.

Friend, I., Y. Landskronner, and E. Losq, 1976, "The Demand for Risky Assets Under Uncertain Inflation," Journal of Finance, 31 (December), 1287-1297.

Green, H., 1964, Aggregation in Economic Analysis: An Introductory Survey (Princeton: Princeton University Press).

Jones, D., 1979, A Structural Econometric Model of the United States Equity Market, Ph.D. Thesis, Harvard University.

, 1980, "Consistent Simple Sum Aggregation over Assets," National Bureau of Economic Research Working Paper No. 573.

Lintner, J., 1972, "Equilibrium in a Random Walk and Log Normal Securities Market," Harvard Institute for Economic Research Discussion Paper No. 235.

Merton, R., 1973, "An Intertemporal Capita1 Asset Pricing Mode1," Econometrica, 41 (September), 867-887. 
APPENDIX

Proof of Theorem 1:

Necessity: Problem (3) can be rewritten in terms of partitioned matrices as:

$$
\begin{array}{ll}
\operatorname{maximize} & \sum_{i=1}^{s}\left[\underline{h}_{i} \underline{\underline{r}}_{i}-(\rho / 2) \underline{h}_{i}{ }^{T_{\Omega_{i}}}{ }_{i} \underline{h}_{i}\right]-(\rho / 2) \sum_{i=1}^{s} \sum_{j \neq i} \underline{h}_{i}{ }^{T} \Omega_{i, j} \underline{h}_{j} \\
\underline{h}_{1}, \ldots, \underline{h}_{s} & \sum_{i=1}^{s} \underline{1} \underline{h}_{i}=1
\end{array}
$$

The first order conditions for this problem are:

$$
\begin{aligned}
& \overline{\underline{r}}_{i}-\rho \Omega_{i, i} \underline{h}_{i}-\rho \sum_{j \neq i} \Omega_{i, j} \underline{h}_{j}-\underline{1} \lambda=0 \text { for } i=1, \ldots, s \\
& \sum_{i=1} \underline{I}^{T} \underline{h}_{i}=1
\end{aligned}
$$

where $\lambda$ is the Lagrangian multiplier associated with the balance sheet constraint.

Equations (A2) and (A3) may be solved to obtain the optimal portfolio shares as functions of the $\underline{1}^{T} \underline{h}_{i}^{*}$ :

$$
\underline{h}_{i}^{*}=(1 / \rho) B_{i} \bar{r}_{i}+\left(\underline{1}^{T} \underline{h}_{i}^{*}\right) \underline{b}_{i}-B_{i} \sum_{j \neq i} \Omega_{i, j} \underline{h}_{j}^{*} \text { for } i=1, \ldots, s
$$

where

$$
\begin{aligned}
& \mathrm{B}_{i}=\left[\Omega_{i, i}^{-1}-\left(1 / \underline{1}^{\mathrm{T}} \Omega_{i, i}^{-1} \underline{1}\right) \Omega_{i, i}^{-1} \underline{1} \underline{1}^{\mathrm{T}} \Omega_{i, i}^{-1}\right] \text { and } \\
& \mathrm{b}_{i}=\left(1 / \underline{1}^{\mathrm{T}} \Omega_{i, i}^{-1} \underline{1}\right) \Omega_{i, i}^{-1} \underline{1} .
\end{aligned}
$$

A necessary condition for (weak) efficiency is that for each " $i$ ", $\left(1 / \underline{1}^{T} \underline{h}_{i}^{*}\right) \underline{h}_{i}$ be invariant with respect to all perturbations in the $\overline{\underline{r}}_{j} \quad(j=i)$ which leave $\underline{1}^{T} \underline{h}_{i}^{*}$ unchanged. For simplicity set $i=1$ and define

$$
h \equiv\left[\begin{array}{c}
\underline{h}_{1} \\
\hat{\underline{h}}_{2}
\end{array}\right] ; \underline{\bar{r}} \equiv\left[\begin{array}{c}
\overline{\underline{r}}_{1} \\
\hat{\underline{r}} \\
\underline{\underline{r}}_{2}
\end{array}\right] ; \Omega \equiv\left[\begin{array}{ll}
\Omega_{11} & \hat{\Omega}_{12} \\
\hat{\Omega}_{12}^{T} & \hat{\Omega}_{22}
\end{array}\right] ; \text { and } B \equiv\left[\begin{array}{cc}
B_{11} & \hat{B}_{12} \\
\hat{B}_{12}^{T} & \hat{B}_{22}
\end{array}\right]
$$


From (A4) we have

(A6)

$$
\hat{\mathrm{h}}_{2}^{*}=(1 / p) \hat{\mathrm{B}}_{2} \hat{\overline{\mathrm{r}}}_{2}+\left(\underline{1}^{\mathrm{T}} \underline{\mathrm{h}}_{2}^{*} \hat{\underline{\mathrm{b}}}_{2}-\hat{\mathrm{B}}_{1} \hat{\Omega}_{12}^{\mathrm{T}} \underline{\mathrm{h}}_{1}^{*}\right.
$$

where

$$
\begin{aligned}
& \left.\hat{\mathrm{B}}_{2} \equiv\left[\hat{\Omega}_{22}^{-1}-\left(1 / \underline{1}^{\mathrm{T}} \hat{\Omega}_{22}^{-1} \underline{1}\right) \hat{\Omega}_{22}^{-1} 1\right) \hat{\Omega}_{22}^{-1} \underline{1} \underline{1}^{\mathrm{T}} \hat{\Omega}_{22}^{-1}\right] \text { and } \\
& \hat{\underline{b}}_{2} \equiv\left(1 / \underline{1}^{\mathrm{T}} \hat{\Omega}_{22}^{-1} \underline{1}\right) \hat{\Omega}_{22}^{-1} \underline{1} .
\end{aligned}
$$

Under the above notation we have

$$
B_{1} \sum_{j \neq 1} \Omega \Omega_{1 j} \underline{h}_{j}^{*}=B_{1} \hat{\Omega}_{12} \hat{h}_{2}^{*} \text {. }
$$

This may be inserted into (A4) for $i=1$ to yield:

$$
\begin{aligned}
& \underline{\mathrm{h}}_{1}^{*}=(1 / \rho) \mathrm{B}_{1} \overline{\underline{\mathbf{r}}}+\left(\underline{1}^{\mathrm{T}} \underline{\mathrm{h}}_{1}^{*}\right) \underline{\mathrm{b}}_{1}-\mathrm{B}_{1} \hat{\Omega}_{12} \hat{\mathrm{h}}_{2}^{*} \\
& =(1 / \rho) \mathrm{B}_{1} \underline{\bar{r}}_{1}+\left(\underline{1}^{\mathrm{T}} \underline{\mathrm{h}}_{1}^{*}\right) \underline{\mathrm{b}}_{1}-\mathrm{B}_{1} \hat{\Omega}_{12} \cdot\left[(1 / \rho) \hat{\mathrm{B}}_{2} \hat{\hat{\mathrm{r}}}_{2}+\left(1-\underline{1}^{\mathrm{T}} \underline{\mathrm{h}}_{1}^{*}\right) \hat{\mathrm{b}}_{2}-\hat{\mathrm{B}}_{2} \Omega_{12}^{\mathrm{T}} \underline{\mathrm{h}}_{1}^{*}\right]
\end{aligned}
$$

or

$$
\left(\mathrm{I}+\mathrm{B} \hat{\Omega}_{1} \hat{\mathrm{B}}_{2} \hat{\Omega}_{12}^{\mathrm{T}}\right) \underline{\mathrm{h}}_{1}^{*}=(1 / \rho) \mathrm{B}_{1} \underline{\bar{r}}_{1}+\left(\underline{\mathrm{T}}^{\mathrm{h}} \underline{\mathrm{h}}_{1}^{*} \underline{\mathrm{b}}_{1}-(1 / \rho) \mathrm{B}_{1} \hat{\Omega}_{12} \hat{\mathrm{B}}_{2} \hat{\overline{\mathrm{r}}}_{2}-\left(1-\underline{1}^{\mathrm{T}} \underline{\mathrm{h}}_{1}^{*}\right) \mathrm{B}_{1} \Omega_{1} \underline{\mathrm{b}}_{2}\right. \text {. }
$$

Thus, a necessary condition for (weak) efficiency is that the quantity

$$
\mathrm{B}_{1} \hat{\Omega}_{12} \hat{\mathrm{B}}_{2} \hat{\overline{\mathrm{r}}}_{2}
$$

be invariant with respect to all perturbations of $\underline{\hat{r}}_{2}$ which leave $\left(\underline{1}^{\mathrm{T}} \underline{h}_{1}^{*}\right)$

unchanged. From (5) it is seen that perturbing $\underline{\hat{\vec{x}}}_{2}$ by $\underline{x}$ leaves $\left(\underline{1}^{\mathrm{T}} \underline{\mathrm{h}}_{1}^{*}\right)$ invariant if and only if

$$
\underline{I}^{\mathrm{T}} \hat{\mathrm{B}}_{22} \underline{\mathrm{x}}=0 \text {. }
$$

The set of $\underline{x}^{\prime} s$ satisfying this relation are the vectors in the column space of the matrix

$$
\left[\mathrm{I}-\left(1 / \underline{1}^{\mathrm{T}} \hat{\mathrm{B}}_{22}^{2} \underline{1}\right) \hat{\mathrm{B}}_{22} \underline{\underline{1}} \underline{1}^{\mathrm{T}} \hat{\mathrm{B}}_{22}\right] .
$$

Thus, if we are to have

$$
\mathrm{B}_{1} \hat{\Omega}_{12} \hat{\mathrm{B}}_{2} \hat{\bar{r}}_{2}=\mathrm{B}_{1} \hat{\Omega}_{12} \hat{\mathrm{B}}_{2}\left(\underline{\bar{r}}_{2}+\underline{\mathbf{x}}\right)
$$

for all $\underline{x}$ satisfying (All), then it must be true that

$$
\left[\left(1 / \underline{1}^{\mathrm{T}} \hat{\mathrm{B}}_{22}^{2} \underline{1}\right) \hat{\mathrm{B}}_{22} \underline{\underline{1}} \underline{1}^{\mathrm{T}} \hat{\mathrm{B}}_{22}\right] \cdot\left[\mathrm{B}_{1} \hat{\Omega}_{12} \mathrm{~B}_{2}\right]^{\mathrm{T}}=\left[\mathrm{B}_{1} \hat{\Omega}_{12} \mathrm{~B}_{2}\right]^{\mathrm{T}} \text {. }
$$


However, since the first matrix in square brackets on the left-hand side of this expression is symmetric and indempotent (i.e., an orthogonal projection matrix), this implies that

(A15) $\quad \mathrm{B}_{1} \hat{\Omega}_{12} \hat{\mathrm{B}}_{2}=\underline{z} \underline{1}^{\mathrm{T}} \hat{\mathrm{B}}_{22}$

for some vector $\underline{z}$. Also, it is easily shown that, since the rank of $B$ is "k-1," $\hat{B}_{22} \underline{1 \neq} \underline{0}$. Hence, upon postmultiplying (A15) by 1 and noting that $\hat{B}_{2} \underline{1}=\underline{0}$ we obtain

(A16) $\underline{0}=\underline{z}$.

Thus, (A17) $\quad \mathrm{B}_{1} \hat{\Omega}_{12} \hat{\mathrm{B}}_{2}=0$.

From the structures of $B_{1}$ and $\hat{B}_{2}$, it is clear that all $\hat{\Omega}_{12}$ satisfying (A17) must be of the form

$$
\hat{\Omega}_{12}=\hat{\underline{c}}_{1} \underline{1}^{\mathrm{T}}+\underline{1}_{\hat{c}^{2}}^{\mathrm{T}}
$$

for some vectors $\hat{c}_{1}$ and $\hat{c}_{2}$.

Since our original choice of $i=1$ was arbitrary, conditions analogous to (A18) must hold for values of "i" different from unity. These conditions when taken together imply that each $\Omega_{i, j}$ takes the form

$$
\Omega_{i, j}=\underline{c}_{i} \underline{1}^{T}+\underline{1}_{j}{ }^{T} ; i \neq j ; i, j=1, \ldots, s
$$

for some fixed vectors $\underline{c}_{i}$ and $\underline{c}_{j}$. This is condition (9) in the statement of the Theorem.

Sufficiency: Sufficiency follows upon noting that if (9) holds, then (A20) $\quad\left(1 / \underline{1}^{\mathrm{T}_{h}} \underline{h}_{i}^{*}\right) \underline{h}_{i}^{*}=\left(\frac{1}{\rho \cdot \underline{1}^{\mathrm{T}} \underline{h}_{i}^{*}}\right) \mathrm{B}_{i} \bar{r}_{i}+\underline{b}_{i}-\frac{\left(1-\underline{1}^{\mathrm{T}} \underline{h}_{i}^{*}\right) \mathrm{B}_{i} \mathrm{c}}{\underline{1}_{i} \underline{h}_{i}^{*}}$

for each "i." Because $\underline{c}_{i}$ is a constant vector, this implies that f-functions satisfying (8) exist where $\underline{f}_{i}$ is given by the right-hand side of (A20).

From (A20), it is also apparent why strong efficiency cannot obtain 
if the $\underline{\underline{r}}$ vector is unrestricted in $R^{k}$ and $k>s>1$. If strong efficiency obtained then it would be necessary that the right-hand side of (A20) be independent of $\overline{\underline{r}}_{j}$ for all " $i$ " and " $j$ " $(\neq i)$. This is impossible if the $\overline{\underline{r}}_{j}$ are unrestricted since by suitably choosing the $\underline{\underline{r}}_{j}, \underline{1}^{T} \underline{h}_{i}^{*}$ can be made to achieve any desired value.

Q.E.D. 